\title{
NRF2-mediated Notch pathway activation enhances hematopoietic reconstitution following myelosuppressive radiation
}

\author{
Jung-Hyun Kim, ${ }^{1}$ Rajesh K. Thimmulappa, ${ }^{1}$ Vineet Kumar, ${ }^{2}$ Wanchang Cui,, ${ }^{3}$ \\ Sarvesh Kumar, ${ }^{1}$ Ponvijay Kombairaju, ${ }^{1}$ Hao Zhang, ${ }^{4}$ Joseph Margolick, ${ }^{4}$ \\ William Matsui, ${ }^{5}$ Thomas Macvittie, ${ }^{3}$ Sanjay V. Malhotra, ${ }^{2}$ and Shyam Biswal ${ }^{1}$

\begin{abstract}
${ }^{1}$ Department of Environmental Health Sciences, Johns Hopkins Bloomberg School of Public Health, Baltimore, Maryland, USA ${ }^{2}$ Laboratory of Synthetic Chemistry, Leidos Biomedical Research, Inc., Frederick National Laboratory for Cancer Research, Frederick, Maryland, USA. ${ }^{3}$ Department of Radiation Oncology, School of Medicine, University of Maryland, Baltimore, Maryland, USA.

${ }^{4}$ Department of Microbiology and Molecular Immunology, Johns Hopkins Bloomberg School of Public Heath, Baltimore, Maryland, USA. ${ }^{5}$ Department of Oncology, Sidney Kimmel Comprehensive Cancer Center, School of Medicine, Baltimore, Maryland, USA.
\end{abstract}

\begin{abstract}
A nuclear disaster may result in exposure to potentially lethal doses of ionizing radiation (IR). Hematopoietic acute radiation syndrome (H-ARS) is characterized by severe myelosuppression, which increases the risk of infection, bleeding, and mortality. Here, we determined that activation of nuclear factor erythroid-2-related factor 2 (NRF2) signaling enhances hematopoietic stem progenitor cell (HSPC) function and mitigates IR-induced myelosuppression and mortality. Augmenting NRF2 signaling in mice, either by genetic deletion of the NRF2 inhibitor Keap1 or by pharmacological NRF2 activation with 2-trifluoromethyl-2'-methoxychalone (TMC), enhanced hematopoietic reconstitution following bone marrow transplantation (BMT). Strikingly, even 24 hours after lethal IR exposure, oral administration of TMC mitigated myelosuppression and mortality in mice. Furthermore, TMC administration to irradiated transgenic Notch reporter mice revealed activation of Notch signaling in HSPCs and enhanced HSPC expansion by increasing Jagged1 expression in BM stromal cells. Administration of a Notch inhibitor ablated the effects of TMC on hematopoietic reconstitution. Taken together, we identified a mechanism by which NRF2-mediated Notch signaling improves HSPC function and myelosuppression following IR exposure. Our data indicate that targeting this pathway may provide a countermeasure against the damaging effects of IR exposure.
\end{abstract}

\section{Introduction}

After an acute radiation disaster caused by either a nuclear power plant accident, a possible terrorist attack, or nuclear warfare, the first responders and civilians are at high risk of exposure to lethal doses of ionizing radiation (IR). The hematopoietic system is highly sensitive to IR, and in particular, IR doses beyond 2 Gy can lead to myelosuppression that is characterized by neutropenia, lymphocytopenia, and thrombocytopenia. Collectively, hematopoietic acute radiation syndrome (H-ARS) increases the risk of infection, bleeding, and death (1-9). There is an unmet need for effective interventions to mitigate the progression of H-ARS after IR exposure (10).

Maintenance of hematopoietic homeostasis and reconstitution after myeloablation relies solely on the self-renewal capability of hematopoietic stem progenitor cells (HSPCs). HSPC self-renewal is regulated by both intrinsic and extrinsic signals that are governed by BM stromal cells, such as osteoblasts and ECs (11). Inhibition of HSPC self-renewal or myelosuppression induced by IR is a direct consequence of DNA damage, leading to apoptosis, senescence, or cell cycle arrest $(2,12,13)$. Antioxidants,

Conflict of interest: Shyam Biswal, Rajesh Thimmulappa, Vineet Kumar, Sanjay Malhotra, and the Johns Hopkins University hold intellectual property on the development of TMC for mitigating radiation injury. Cureveda LLC has licensed this intellectual property. Shyam Biswal and Rajesh K. Thimmulappa are cofounders of, have equity in, and serve as scientific consultants for Cureveda LLC. These potential individual and institutional conflicts of interest have been reviewed and managed by the Johns Hopkins University School of Public Health.

Citation for this article: J Clin Invest. 2014;124(2):730-741. doi:10.1172/JCI70812. antiapoptotic cytokines, or hematopoietic growth factors that could improve HSPC survival, maintenance, and/ or proliferation are being evaluated as radiomitigators (14-16).

Nuclear factor erythroid-2-related factor 2 (NRF2) regulates an adaptive cytoprotective response to counteract the deleterious effects of ROS, such as DNA damage and apoptosis, and confers cytoprotection following exposure to environmental oxidants (17-22). Upon activation, NRF2 dissociates from its cytosolic inhibitor KEAP1 and mediates transcriptional activation of its target genes, which include multiple antioxidants and electrophile detoxification enzymes $(17,19,23)$. The radioprotective role of NRF2 is evident in a wide variety of cells and is mediated by increasing DNA repair responses (20), neutralizing ROS, and reducing apoptosis (21). Recently, we and others (24) have shown that HSPCs isolated from NRF2-deficient ( $\mathrm{Nrf}^{--}$) mice failed to reconstitute following bone marrow transplantation (BMT). Here, we report that oral administration of a pharmacological NRF2 activator improves HSPC function and mitigates myelosuppression and mortality in mice, even 24 hours after total body irradiation (TBI). Importantly, we show for what we believe to be the first time that NRF2 enhances the repopulating capacity of HSPCs in BM by activating Notch signaling via intrinsic and extrinsic mechanisms.

\section{Results}

Activation of NRF by genetic disruption of Keap1 improves the repopulating capacity of HSPCs by intrinsic and extrinsic mechanisms. To address whether augmenting NRF2 activity in hematopoi- 
A

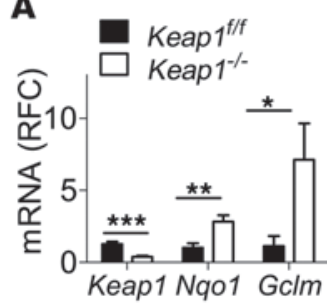

B

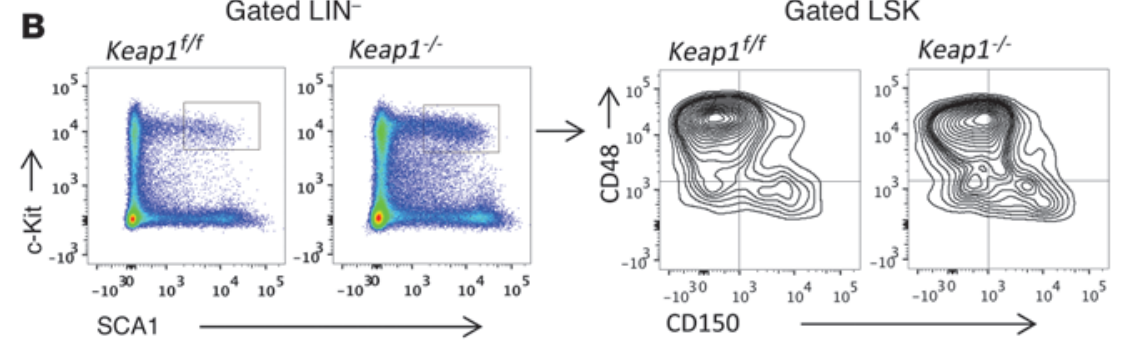

Gated LIN
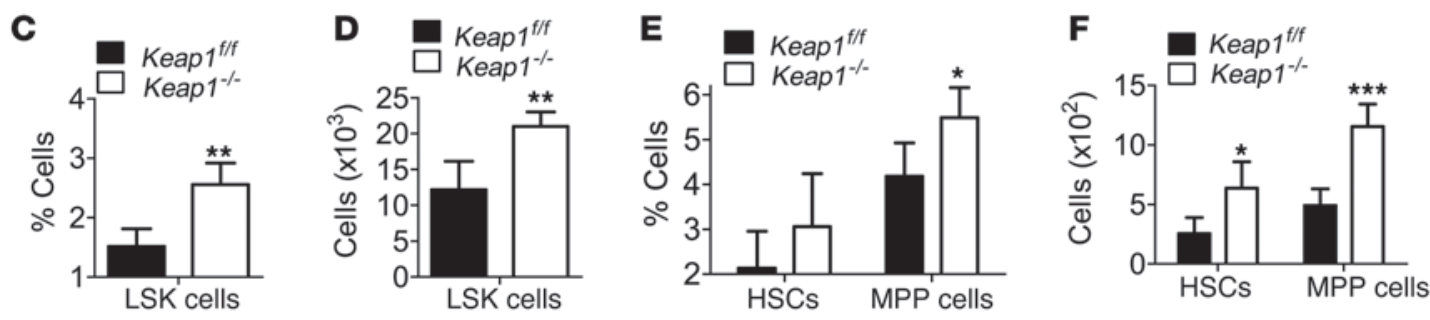

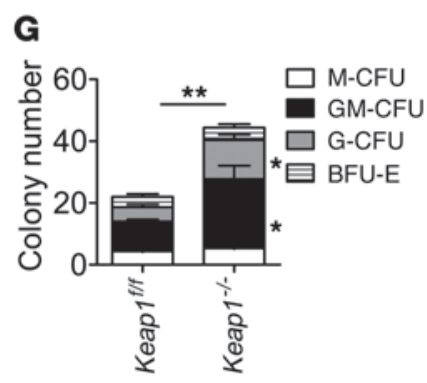

Figure 1

Augmenting NRF2 signaling by genetic disruption of Keap1 improves HSPC function under steady-state conditions. (A) mRNA expression of Keap1 and NRF2-regulated genes Nqo1 and Gclm in BM cells isolated from poly(I:C)-treated MxCre-Keap $1_{\text {flox } / f l o x}$ mice and floxed controls. ${ }^{\star} P<0.05 ;{ }^{*} P<0.01 ;{ }^{* \star} P<0.001$. RFC, relative fold change. (B-F) Flow cytometric analysis (B), percentage (C), and absolute number of LSK cells (D). Percentage (E) and absolute number (F) of HSCs and MPP cells in BM from MxCre-Keap1flox/flox mice and floxed controls following poly $(\mathrm{I}: \mathrm{C})$ treatment under baseline conditions. MxCre-Keap $1^{-/}$is represented as Keap $1^{-/-}$in the graph legends. ${ }^{*} P<0.05$, ${ }^{* *} P<0.01$, and ${ }^{* * *} P<0.001$ compared with respective floxed controls $(n=10-12)$. (G) CFU/200 LSK cells from MxCre-Keap $1^{-1-}$ mice and floxed controls. ${ }^{\star} P<0.05 ;{ }^{* *} P<0.01$. BFU-E, erythroid burst-forming units; M-CFU, megakaryocyte CFU.

etic cells affects HSPC function, we used poly(I:C)-inducible $M x$ Cre-Keap $1^{\text {flox/flox }}$ mice, in which poly(I:C) injection induced

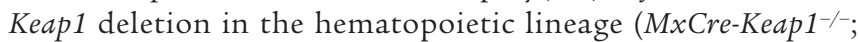
Supplemental Figure 1; supplemental material available online with this article; doi:10.1172/JCI70812DS1). Analysis of hematopoietic cells from BM, defined as $\mathrm{LIN}^{-} \mathrm{SCA}^{+} \mathrm{c}-\mathrm{KIT}^{+}$ cells (LSK cells; highly enriched for long-term and short-term HSCs and multipotent progenitor [MPP] cells; herein referred to as HSPCs) (24), revealed that expression of Keap1 was reduced, while expression of NRF2-regulated antioxidant genes Nqo1

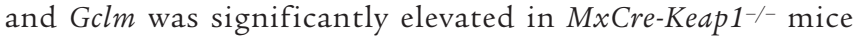
compared with that in floxed controls, demonstrating activation of NRF2 in HSPCs (Figure 1A). Although the antioxidants were elevated, the constitutive levels of ROS in LSK cells from $M x C r e-K e a p 1^{-/-}$and Keap $1^{\text {flox/flox }}$ mice were comparable (Supplemental Figure 2). Examination of peripheral blood (PB) 4 weeks after poly(I:C) treatment revealed no significant increase in total leukocyte counts, but the number of platelets was significantly higher in MxCre-Keap $1^{-/-}$mice compared with that in poly(I:C)treated floxed controls (Supplemental Table 1) under steadystate conditions.
Next, we assessed whether augmenting NRF2 in hematopoietic cells affects hematopoiesis in steady-state conditions. MxCre-Keap $1^{-/-}$mice showed a higher percentage of LSK cells among the total BM cells, which resulted in a 1.7-fold increase in the absolute number of LSK cells compared with that in Keap $1^{\text {flox/flox }}$ mice (Figure 1, B-D). Flow cytometric analysis of LSK cells revealed no significant changes in the frequency of HSCs (CD150 $\left.{ }^{+} \mathrm{CD} 48^{-}\right)$, but a statistically significant increase in the frequency of MPP

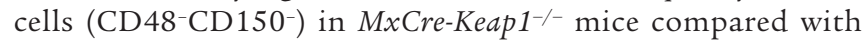
Keap $1^{\text {flox/flox }}$ mice (Figure 1E), resulting in 2.5 and 2.3-fold increases in the absolute number of HSCs and MPP cells, respectively (Figure $1 \mathrm{~F})$. Of note, we did not observe any significant difference in the population of BM stromal cells (identified as CD45-TER119-)

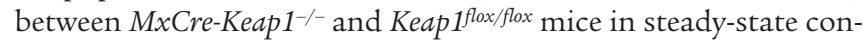
ditions (Supplemental Figure 3, A and B). Next, we assessed the in vitro colony-forming activity of LSK cells to determine whether augmenting NRF2 alters the frequency of committed progenitor cells. We observed a statistically significant increase in total CFU, which was contributed mainly by GM-CFU and CFU granulocytes (G-CFU) in MxCre-Keap1 $1^{-/}$mice compared with that found

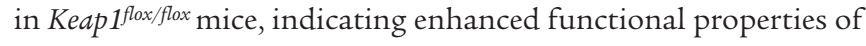


MxCre-Keap1 $1^{-/}$HSPCs (Figure 1G). However, under steady-state conditions in vivo, the number of myeloid progenitors (such as granulocyte-monocyte progenitors [GMPs], common lymphoid progenitors [CLPs], and megakaryocyte erythroid progenitors [MEPs]) was comparable between MxCre-Keap1 $1^{-/-}$and Keap $1^{\text {flox/flox }}$ mice (Supplemental Figure 3, C and D).

To assess HSPC function in vivo, we performed competitive transplantation of LSK cells isolated from MxCre-Keap $1^{\text {flox }}$ flox or Keap $1^{\text {flox/flox }}$ mice 4 weeks after poly(I:C) injection into lethally irradiated WT mice (CD45.1). Eight and 14 weeks after BMT, PB analysis of recipient mice showed greater engraftment of donor cells derived from poly(I:C)-injected $M x C r e-K e a p 1^{\text {flox/flox }}$ mice compared that seen in Keap $1^{\text {flox } / \text { flox }}$ mice (Figure 2, A-C), indicating that gain of NRF2 function in HSPCs improves their short-term repopulating capacity. To further clarify the intrinsic role of NRF2 in HSPCs, we injected primary recipient WT mice (CD45.1) with poly(I:C) after competitive transplantation with LSK cells isolated from

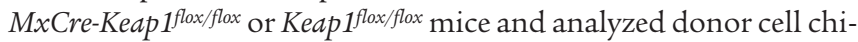
merism. Three weeks after BMT, poly(I:C)-treated recipient mice transplanted with $M x$ Cre-Keap $1^{\text {flox/flox }}$ donor cells showed a higher total number of CD45.2 cells compared with that in poly(I:C)-injected recipient mice transplanted with Keap1 flox/flox donor cells (Figure 2, D and E). Poly(I:C) injection induces type 1 IFN signaling and activates HSC proliferation (25). To exclude any effect of poly(I:C) on our results, we transplanted BM cells from primary transplant recipients into a secondary group of lethally irradiated WT mice. PB analysis showed a higher number of total donor CD45.2 cells and multilineage engraftment, with significantly higher numbers of myeloid lineage $\left(\mathrm{GR} 1^{+}\right)$cells in the secondary recipient mice (CD45.1) that received BM from poly(I:C)-injected primary recipient mice transplanted with $M x C r e-K e a p 1^{\text {flox/flox }} \mathrm{LSK}$ cells compared with those found in Keap $1^{\text {flox/flox }}$ mice (Figure 2, D-F, and Supplemental Figure 4).

HSPCs reside in a specialized microenvironment located in the BM, referred as the "niche," and it has been well demonstrated that BM stromal cells tightly regulate HSPC maintenance and expansion through extrinsic signals $(26,27)$. To gain insight into a possible role of NRF2 in BM stromal cells in the regulation of HSPC function, we used tamoxifen-inducible CMVCre-Keap $1^{\text {flox/flox }}$ mice, in which tamoxifen treatment induced whole-body Keap1 deletion (Supplemental Figure 5A). Tamoxifen-treated CMVCre-Keap $1^{\text {flox/flox }}$ mice showed reduced expression of the Keap1 gene and increased expression of NRF2-regulated genes in whole BM and CD45 stromal cells (Supplemental Figure 5B and Figure 2H) compared with tamoxifen-treated Keap $1^{\text {flox/flox }}$ mice. Similar to the $M x C r e$ model, we observed a significant increase in the frequency of the $\mathrm{LSK}^{-}$cell population in tamoxifen-treated CMVCre-Keap $1^{\text {flox } / f l o x}$ mice compared with that seen in Keap $1^{\text {flox/flox }}$ mice (Supplemental Figure 5C). To address the role of Keap $1^{-1-}$ BM stromal cells in enhancing HSPC function, we cultured WT LSK cells with BM stromal cells isolated from tamoxifen-treated CMVCre-Keap $1^{\text {flox } / f l o x}$

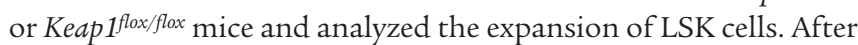
7 days of coculture, the expansion of LSK cells was approximately $50 \%$ higher in CMVCre-Keap1-/- stromal cells than in Keap1 $1^{\text {flox/flox }}$ stromal cells (Figure 2, G-I, and Supplemental Figure 5D). These results suggest that augmenting NRF2 signaling in BM stromal could promote HSPC expansion via extrinsic signals.

Pharmacological activation of NRF2 enhances hematopoietic reconstitution following $B M T$. We next evaluated whether pharmacological activation of NRF2 augments the repopu- lating capacity of HSPCs. We have previously shown that 2-trifluoromethyl-2'-methoxychalcone (TMC), a synthetic chalcone derivative, is a potent small-molecule NRF2 activator (28). Multiple oral administrations of TMC (6 times, once every 48 hours) upregulated NRF2-dependent target genes Nqo1, Gclm, and $\mathrm{Ho1}$ in BM cells and other tissues (such as small intestine) of mice (Supplemental Table 2). A CFC assay showed that administration of TMC (6 times, once every 48 hours) significantly elevated functional hematopoietic progenitor cells in the BM; however, the total number of LSK cells was unaffected compared with that in vehicle-treated mice under steady-state conditions (Supplemental Figure 6, A and B). We also noted that daily oral administration of TMC for 2 weeks in healthy mice did not affect the PB counts and caused no apparent renal or hepatic toxicity (Supplemental Table 3). To determine whether TMC administration promoted HSPC function in vivo, we transplanted WT BM cells (CD45.2) into lethally irradiated WT mice (CD45.1) and treated the recipient mice orally with TMC or vehicle 6 times following BMT (initiated 1 hour after BMT, and then once every 48 hours). Two weeks after BMT, the donor cell engraftment was approximately $50 \%$ and approximately $400 \%$ higher in PB (Figure 2J) and $\mathrm{BM}\left(\mathrm{LIN}^{-}\right.$cells; Figure $\left.2 \mathrm{~K}\right)$, respectively, in recipient mice treated with TMC compared with those treated with vehicle. Taken together, these data suggest that pharmacological activation of NRF2 improves BMT.

Nrf2-null mice are more sensitive to TBI-induced mortality and myelosupression. The ability of NRF2 to increase hematopoietic reconstitution led us to hypothesize that pharmacological activation of NRF2 mitigates TBI-induced myelosuppression. To test this hypothesis, we used $\mathrm{Nrf2}^{-/-}$mice to determine whether NRF2 is a critical host factor for survival after TBI. In response to increasing doses of TBI, Nrf2 $2^{-/-}$mice exhibited greater mortality (Figure 3, A and B) compared with $\mathrm{Nrf2} 2^{+/+}$mice. CFC assays showed greater reductions in functional hematopoietic progenitor cells in $\mathrm{Nrf2} 2^{-/-}$mice than in $\mathrm{Nrf2}^{+/+}$mice 48 hours after TBI

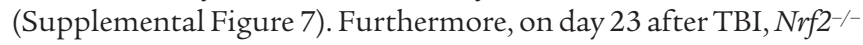
mice had a lower blood cell count (Figure 3C) and BM LSK cell population (Figure $3 \mathrm{D}$ ) than $\mathrm{Nrf2^{+/+ }}$ mice. Collectively, these results suggest that NRF2 is essential for protection against IR-induced myelosupression and mortality.

TMC treatment mitigates TBI-induced mortality and myelosupression in an NRF2-dependent manner. Next, we addressed whether augmenting NRF2 signaling with a pharmacological activator mitigates TBI-induced mortality. First, we sought to determine the baseline NRF2 activity in HSCs and BM stromal cells in mice after IR and whether TMC administration increases NRF2 activity in these BM compartments. NRF2 activity, as indicated by Nqo1 and Gclm expression, was markedly reduced in $\mathrm{CD} 45^{+} \mathrm{LIN}^{-}$(enriched for HSPC populations) and CD34- LSK cells on days 1 and 2 and recovered to normal levels by day 12 following TBI. Similarly, NRF2 activity in BM stromal cells (CD45-TER119-) was significantly reduced on days 1 and 2, but was markedly increased by day 12 after TBI. However, oral administration of TMC every other day for a total of 6 doses, beginning 24 hours after TBI, significantly increased NRF2 activity in HSPCs and BM stromal cells compared with that observed in the vehicle-treated group (Figure 4, A-C). Of note, we did not observe any change in expression of the $\mathrm{Nrf} 2$ gene following radiation exposure (Figure 4, A-C), suggesting that the regulation of NRF2 activity is posttranscriptionally mediated (enhanced protein stability), as reported previously (19, 29-33). 
A

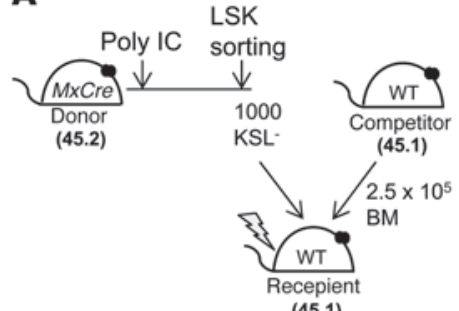

(45.1)

D

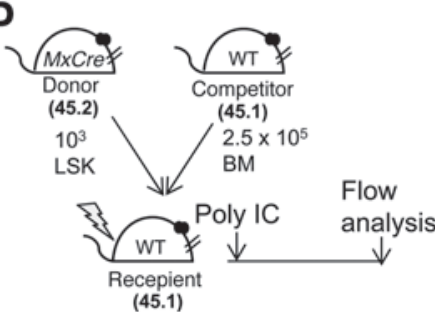

B

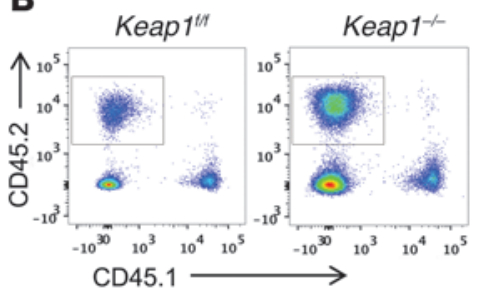

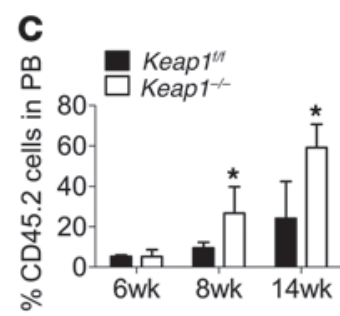

G
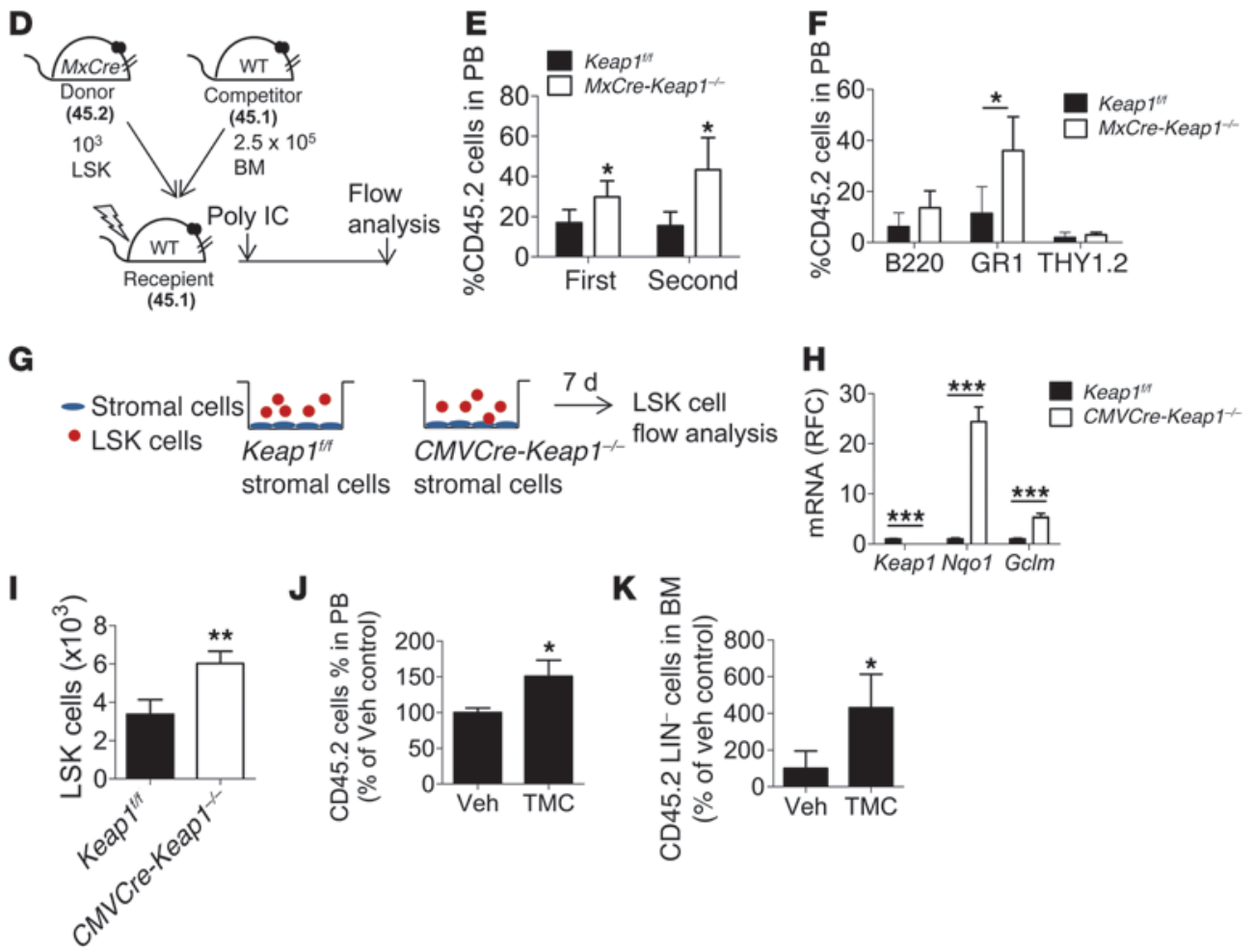

$\mathbf{K} \sum_{\text {m }}$

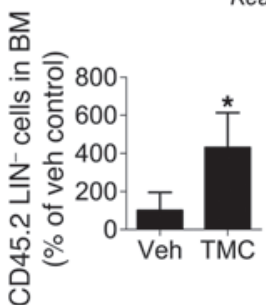

Figure 2

Augmenting NRF2 signaling enhances BM engraftment. (A) Schematic of experiment depicted in B and C. Sorted LSK cells from poly(I:C)-treated MxCre-Keap $1^{\text {flox/flox }}$ or floxed controls were transplanted into recipient mice with 250,000 competitor cells. (B and C) Flow cytometric analysis (B) and percentage of donor cell in PB at 6, 8, and 14 weeks following BMT (C). (D) Schematic of experiment depicted in E and $\mathbf{F}$. Mice were treated daily with poly $(\mathrm{I}: \mathrm{C})$ for 7 days after BMT. (E) Percentage of donor cells in PB following serial BMT (first and second BMT). (F) Donor-derived granulocyte $\left(\mathrm{GR} 1^{+}\right)$, B cell $\left(\mathrm{B} 220^{+}\right)$, and T cell $\left(\mathrm{THY} 12^{+}\right)$lineages in PB of recipient mice after the second transplantation. (G) Schematic of ex vivo experiment depicted in $\mathbf{H}$ and I. BM stromal cells isolated from tamoxifen-treated CMVCre-Keap ${ }^{\text {flox/flibx }}$ or floxed mice were plated as feeder cells. Five hundred sorted LSK cells were cocultured with BM stromal cells. LSK progeny cells harvested on day 7 were stained and analyzed. (H) Expression of Keap1, Nqo1, and Gclm genes in BM stromal cells (CD45-TER119-) isolated from CMVCre-Keap1-/mice and floxed control mice. (I) Total number of LSK cells on day 7. ( $\mathbf{J}$ and $\mathbf{K}$ ) Percentage of donor cells (CD45.2) in the PB (J) and BM (LIN-) (K) of TMC- or vehicle-administered recipient mice ( $n=10-15 /$ group) after BMT. CD45.2 BM cells (500,000 cells) were transferred into irradiated recipient WT mice. The recipient mice were orally administered vehicle (Veh) or TMC 6 times, once every 48 hours, beginning 1 hour after BMT ( $n=10-15$ group). ${ }^{*} P<0.05,{ }^{* *} P<0.01,{ }^{* *} P<0.001$.

To test whether pharmacological activation of NRF2 mitigates radiation-induced mortality, mice were exposed to a range of TBI doses (6.9 Gy [LD $\left.\mathrm{LD}_{30 / 30}\right], 7.1 \mathrm{~Gy}\left[\mathrm{LD}_{70 / 30}\right]$, and $\left.7.3 \mathrm{~Gy}\left[\mathrm{LD}_{100 / 30}\right]\right)$ and were administered TMC or vehicle orally every other day for a total of 6 doses, beginning 24 hours after TBI. Then, we monitored the survival rate. Relative to vehicle, TMC administration dramatically improved the 30-day survival rate following 6.9 Gy (30\% vehicle vs. 90\% TMC; Figure 4D) and $7.1 \mathrm{~Gy}(0 \%$, vehicle vs. 70\%, TMC; Figure 4E) TBI doses. At a 7.3-Gy TBI dose, TMC administration failed to improve survival when administered 24 hours after IR; however, TMC significantly improved survival rates to $60 \%$ and $50 \%$ when started 1 hour and 6 hours after TBI, respectively (Figure 4F). We found that TMC administration 48 hours after a 6.9-Gy TBI dose failed to improve survival (Supplemental Figure 8). Furthermore, we observed that TMC administration failed to improve survival rates in $\mathrm{Nrf2^{-/- }}$ mice following $6.9 \mathrm{~Gy} \mathrm{TBI}$, indicating that its specific mechanism of action is dependent on NRF2 activation (Figure 4G).

We validated the radiomitigative efficacy of TMC in the CD1 mouse strain. TMC administration 24 hours after TBI significantly 

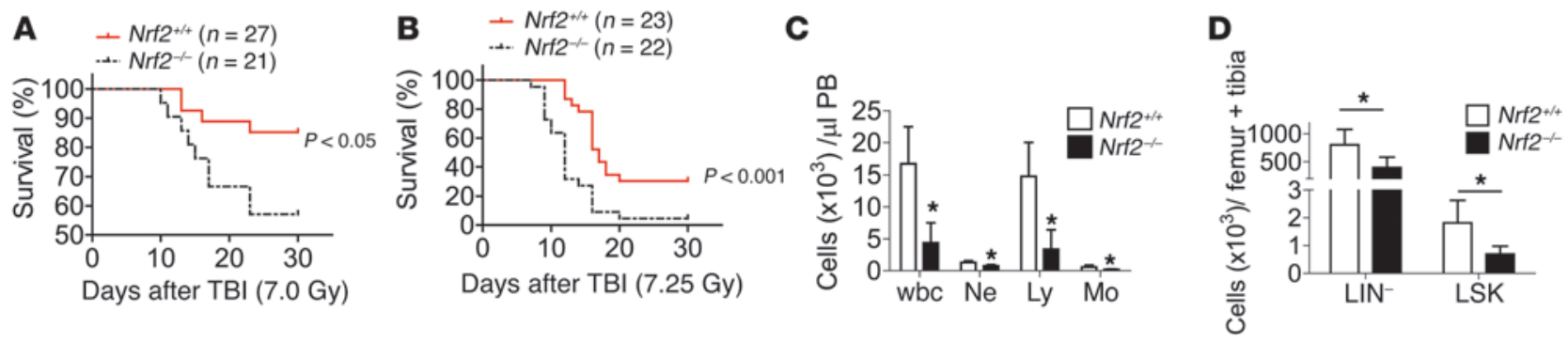

\section{Figure 3}

NRF2-null mice show increased sensitivity to TBI-induced mortality and myelosupression. (A and B) Thirty-day survival rate of $\mathrm{Nrf2}^{-/-}$and $\mathrm{Nrf2}^{+/+}$ mice exposed to (A) 7.0 Gy and (B) 7.25 Gy TBI. (C and D) Differential blood counts (C) (wbc, neutrophils [Ne], lymphocytes [Ly], and monocytes [Mo]) and LSK cell populations (D) in $\mathrm{Nrf2}^{+/+}$and $\mathrm{Nrf2}^{-/-}$mice on day 23 after TBI (6.9 Gy). ${ }^{*} P<0.05$ compared with $\mathrm{Nrf2} 2^{-/-}$mice.

increased survival of CD1 mice compared with vehicle-treated mice (Supplemental Figure 9). To verify that the radiomitigative potency of targeting the NRF2 pathway is not specific to TMC, we used another pharmacological activator of NRF2, CDDO-methyl ester (CDDO-Me, 2-cyano-3,12-dioxooleane-1,9(11)-dien-28-oic acid methyl ester) (34-36). CDDO-Me is a synthetic triterpenoid that was under phase III clinical development for the treatment of advanced chronic kidney disease $(37,38)$ However, due to adverse events in the phase III clinical trial, further development of CDDO-Me was terminated (39). Like TMC, we found that oral administration of CDDO-Me 24 hours after TBI $\left(\mathrm{LD}_{30}\right.$ or $\mathrm{LD}_{100}$ dose) significantly increased NRF2 target gene expression (Supplemental Table 4) and improved the survival of C57BL/6 mice (Supplemental Figure 10, A and B) in an NRF2-dependent manner (Supplemental Figure 10C).

Next, we investigated whether TMC affected hematopoietic recovery in mice exposed to $6.9 \mathrm{~Gy}$ TBI. The decline in $\mathrm{PB}$ counts was similar in TMC- and vehicle-administered mice until day 12 after TBI (Figure 5A). However, TMC enhanced recovery of all PB cell lineages (neutrophils, monocytes, lymphoid cells, erythrocytes, and platelets) compared with those in vehicle-treated mice (Figure 5, A-C) from days 15 to 23 after radiation. Of note, oral administration of CDDO-Me 24 hours after TBI also showed enhanced recovery of all PB cell lineages after TBI (Supplemental Figure 11).

Concurrently, histological analysis of femurs on day 23 showed markedly higher BM cellularity in TMC-administered mice, compared with vehicle-treated mice (Figure 5D and Supplemental Figure 12). Consistent with the higher neutrophil blood count, blood bacteremia, as assessed by blood cultures, was significantly reduced in TMC-administered mice compared with that in vehicle-treated mice (Figure 5E). These results confirm that TMC treatment mitigated TBI-induced mortality and myelosuppression in mice.

TMC treatment enhanced HSPC regeneration in irradiated mice. We analyzed the HSPC population in the BM of TMC- and vehicle-administered, irradiated mice. The number of HSC $\left(\mathrm{LSK}^{+} \mathrm{CD} 48^{-} \mathrm{CD} 150^{+}\right)$and MPP cells $\left(\mathrm{LSK}^{+} \mathrm{CD} 48^{-} \mathrm{CD} 150^{-}\right)$ decreased markedly by day 3 after TBI. However, TMC-treated mice showed a marked increase in the recovery of LSK, HSC, and MPP cell populations compared with that observed in vehicle-treated mice (Figure 6A) on day 21 after IR. This increase in HSPCs by day 21 corresponded with an approximate 3 -fold increase in $\mathrm{KI}_{6} 7^{+}$cells, which are markers of proliferation, in the
TMC-treated group compared with that observed in the vehicle-treated group (Figure 6B). To determine whether TMC treatment mediated radioprotection of HSPCs, we measured ROS levels, glutathione levels, and live and dead cells on day 3 after TBI. Although, TMC increased glutathione and reduced ROS in LSK cells (Figure 6C), the percentage of viable LSK cells on day 3 following TBI was elevated only modestly compared with that in vehicle-treated mice (Supplemental Figure 13A). Also, there was no significant difference in the number of dead (Supplemental Figure 13B) or apoptotic LSK cells between vehicle- and TMCtreated mice on day 3 after TBI (data not shown). These results suggest that TMC-mediated hematopoietic reconstitution in the irradiated mice was not mainly due to protection from radiation-induced cytotoxicity, but rather was a result of an augmented proliferation of the HSPC population. To further elucidate how TMC administration increased functional HSPC populations in irradiated mice, we performed a competitive repopulation assay (as shown in Figure 6D). At 6 weeks, the recipient mice transplanted with BM cells from TMC-administered irradiated mice showed a 4-fold increase in the total number of CD45.2 cells and a significantly higher number of granulocytes $\left(\mathrm{GR} 1^{+}\right)$, B cells $\left(\mathrm{B} 220^{+}\right)$, and T cells (THY1.1 $1^{+}$in PB compared with those found in vehicle-treated controls (Figure 6, E-G).

Augmenting NRF2 enhances HSPC expansion by upregulating Notch signaling. We next addressed how augmenting NRF2 signaling increases HSPC expansion in irradiated mice. By integrating global ChIP sequencing (ChIP-Seq) and transcriptomic profiles from cell models containing gain and loss of NRF2 function, we recently identified Jagged 1 (JAG1), a ligand for Notch1, as a direct transcriptional target of NRF2 (40-42) in embryonic fibroblast cells. Previous studies have shown that activation of Notch signaling enhances HSC self-renewal, while its loss impairs the maintenance of HSCs (43-48). Hence, we hypothesized that augmenting NRF2 could enhance HSPC expansion by activating Notch signaling. To address this, we first measured the expression of Notch1 and Notch target genes in BM cells after activating NRF2 signaling. BM cells from MxCre-Keap1 ${ }^{-1-}$ mice showed greater expression of Notch1 and the Notch target gene Hes1 compared with that seen in floxed controls (Figure 7A). Following TBI, TMC-treated mice showed greater expression of Notch1 and Hes1 in BM cells compared with vehicle-treated mice (Figure 7B). In addition, compared with vehicle, TMC administration markedly upregulated Jag1 (Figure 7C), but not the expression of Jag2 or delta-like 4 (Supplemental Fig- 
A $\mathrm{CD}^{-} 5^{+}$Lineage $-\mathrm{HSPC}$

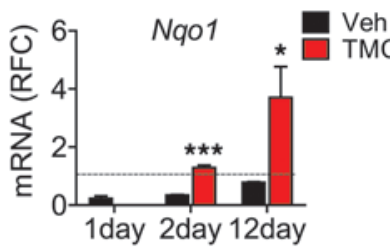

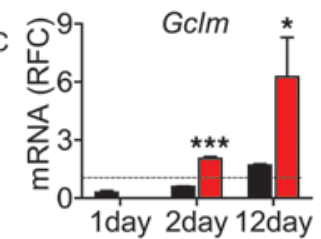

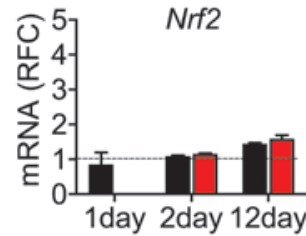

\section{C $\mathrm{CD}^{-} 4^{-} \mathrm{LSK}$}

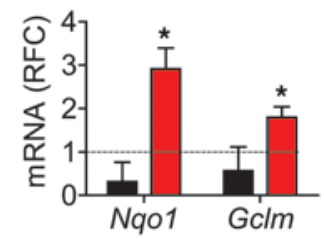

B CD45 stromal

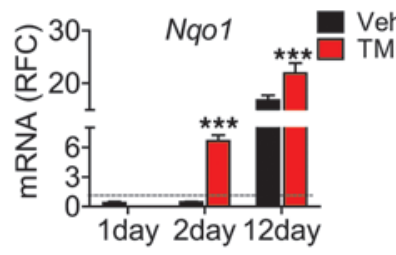

D

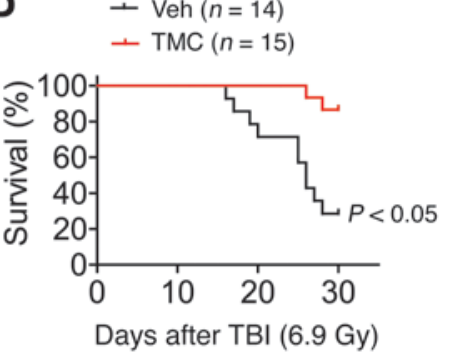

$\mathbf{F}$

- Veh, after $24 \mathrm{~h}(n=20)$

- TMC, after $1 \mathrm{~h}(n=20)$

- TMC, after $6 \mathrm{~h}(n=20)$

- TMC, after 24h $(n=20)$

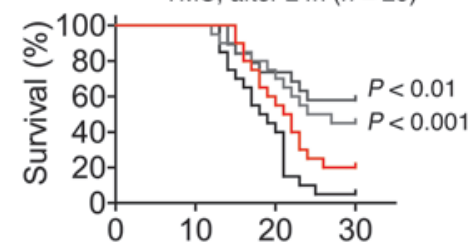

Days after TBI (7.3 Gy)
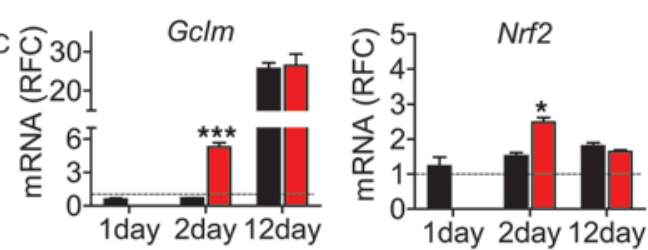

E $\quad-\operatorname{Veh}(n=14)$

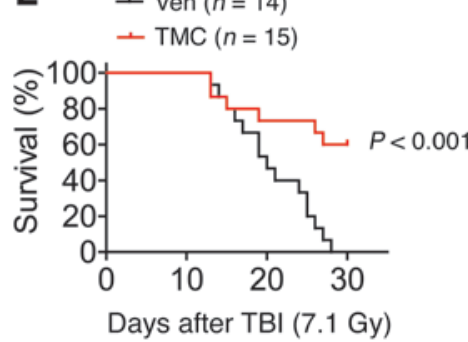

G

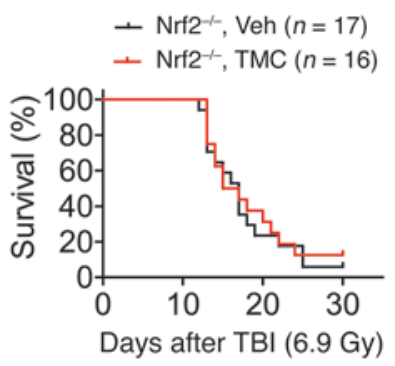

\section{Figure 4}

Administration of NRF2 activator TMC mitigates TBI-induced mortality in mice in an NRF2-dependent manner. (A-C) Relative gene expression of Nq01, Gclm, or Nrf2 in (A) CD45+LIN- HSPCs, (B) CD45 stromal cells, and (C) CD34- LSK cells isolated from BM of irradiated mice. Mice were treated with vehicle or TMC 24 hours after TBI and every 48 hours after 6.9 Gy radiation. ${ }^{*} P<0.05$; ${ }^{\star \star \star} P<0.001$. (D and $\mathbf{E}$ ) Thirty-day survival rates of mice administered TMC or vehicle 6 times (once every 48 hours), beginning 24 hours after 6.9 Gy (D) and 7.1 Gy (E) TBI. (F) Thirty-day survival rates of mice administered TMC or vehicle 6 times (once every 48 hours), beginning 1, 6, or 24 hours after 7.3 Gy TBI. (G) Thirty-day survival rates of $\mathrm{Nrf2}^{-/-}$ mice administered TMC or vehicle 6 times (once every 48 hours), beginning 24 hours after 6.9 Gy TBI. ure 14, A and B), in BM cells, and this upregulation peaked on day 7 following TBI. Next, to confirm that NRF2 activates Notch signaling in HSPCs, we used transgenic Notch reporter (TNR) mice, in which GFP is induced by Notch activation (49). We found that TMC administration to unirradiated TNR mice significantly increased the percentage of GFP-positive LSK cells and colonyforming activity, but did not alter the total number of LSK cells in the BM as compared with that in vehicle-treated mice (Supplemental Figure 15, A-C). In contrast, TMC administration to TNR mice following TBI increased the expression of GFP in bone sections and the frequency of GFP-positive LSK cells compared with vehicle-treated mice (Figure 7, D and E), indicating an upregulation of Notch signaling. Finally, to determine whether Notch signaling is involved in mediating hematopoietic reconstitution following TMC administration, we cotreated vehicle- or TMC-administered irradiated mice with or without the Notch inhibitor $\gamma$ secretase inhibitor II (GSI) (44). On day 12, we found that the population of LSK cells was significantly higher in TMC-treated irradiated mice compared with that in vehicle-treated mice (Figure 7F). However, GSI cotreatment abrogated the ability of TMC to repopulate the LSK cells in irradiated mice. Collectively, these findings confirm that TMC mediates hematopoietic reconstitution by activating the Notch signaling pathway.

TMC enhances HSPC expansion by upregulating Jag1 in BM stromal cells in vitro. JAG1 expressed by the BM stromal cells interacts with the Notch receptor and induces survival and expansion of HSPCs in vitro and in vivo $(50,51)$. We found that in vitro TMC treatment increased Nqo1, Gclm, and Jag1 mRNA expression in BM stromal cells isolated from unirradiated (Figure $8, A$ and B) and irradiated (data not shown) $\mathrm{Nrf2} 2^{+/+}$mice, but not in those from $\mathrm{Nrf2} 2^{-/-}$mice. To show that this elevated JAG1 expression is partly responsible for the NRF2-mediated increase in HSPC repopulation, BM stromal cells from irradiated $\mathrm{Nrf2} 2^{+/+}$or $\mathrm{Nrf2}^{-/-}$mice were exposed to TMC or vehicle ex vivo for 12 hours and subsequently cocultured with irradiated LSK cells. The expansion of LSK cells was markedly (2-fold) elevated in TMC-treated $\mathrm{Nrf2}^{+/+} \mathrm{BM}$ stromal cells 
A

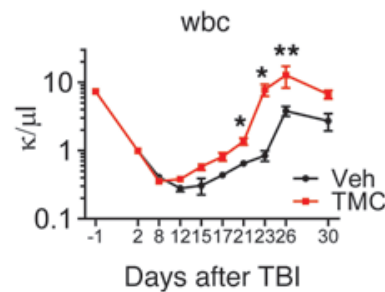

Days after TBI

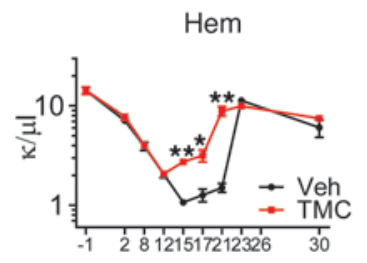

Days after TBI
PIt

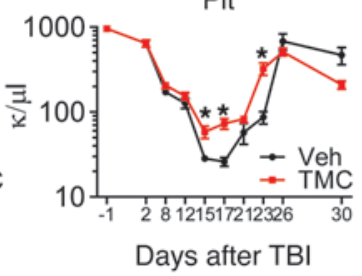

rbc

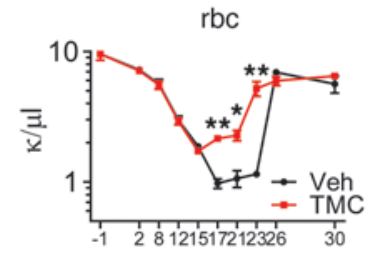

Days after TBI
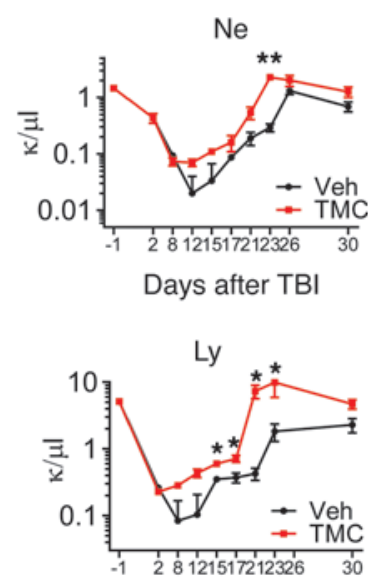

Days after TBI

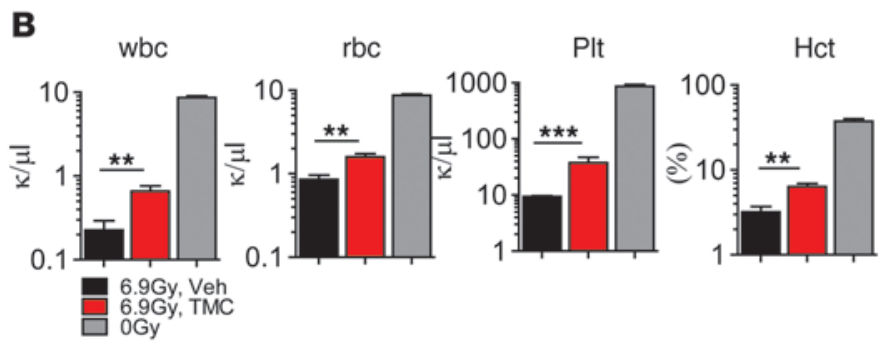

D
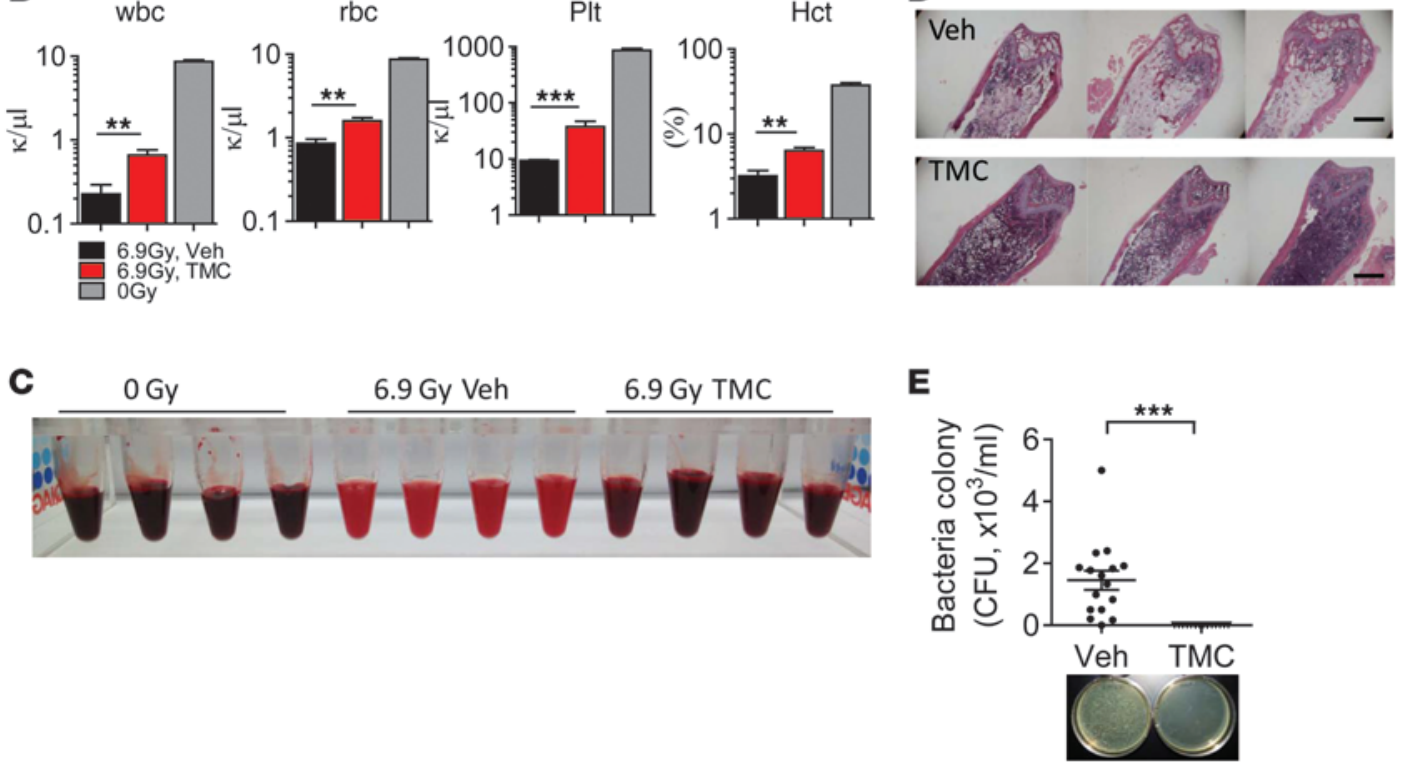

Figure 5

Administration of NRF2 activator TMC mitigates TBI-induced myelosuppression in mice. Mice exposed to 6.9 Gy TBI were administered 6 doses of vehicle or TMC (once every 48 hours), beginning 24 hours after TBI. At each time period indicated, a cohort of mice $(n=5-8)$ were sacrificed and PB was isolated. (A and B) Differential blood cell counts (wbc, rbc, neutrophils, platelets [PIt], and hematocrit [Hct]) in TMC- or vehicle-administered mice at the indicated time period $(\mathbf{A})$ and on day 23 after TBI $(\mathbf{B})$. ${ }^{\star} P<0.05 ;{ }^{\star \star} P<0.01 ;{ }^{* \star} P<0.001$. Data are representative of two independent experiments. (C) Image of blood samples isolated from TMC- or vehicle-treated mice on day 23 following TBI. (D) H\&E-stained bones of TMC- or vehicle-administered mice on day 23 after TBI. Scale bars: $1 \mathrm{~mm}$. (E) Analysis and image of bacterial CFU in PB of TMC- or vehicle-administered mice on days 18-23 after TBI $(n=16-25) .{ }^{* *} P<0.001$ compared with vehicle-treated mice.

compared with the vehicle-treated cells (Figure 8C), while blocking JAG1 binding to the Notch receptor by the anti-JAG1 antibody inhibited LSK cell expansion. In contrast, TMC-treated Nrf2 $2^{-/-} \mathrm{BM}$ stromal cells did not induce HSPC expansion compared with vehicle-treated cells (Figure 8D). Taken together, these results suggest that activation of NRF2 by TMC increases Notch signaling, partly by upregulating JAG1, and enhances HSPC repopulation through cooperation with BM stromal cells.

\section{Discussion}

Regulators of ROS, cell cycle, and apoptosis play important roles in HSC self-renewal and maintenance under homeostatic condi- tions and in responses to genotoxic stressors such as radiation $(2,14,43,52,53)$. NRF2 regulates diverse cellular processes, which include redox regulation, apoptosis, and proliferation, by inducing a broad spectrum of cytoprotective proteins $(19,54)$. Previously, using NRF2-null mice, we reported that NRF2 is essential for regulating HSPC function and hematopoiesis (24). More recently, Tsai et al. (55) also confirmed that loss of NRF2 impairs the repopulating capacity of HSPCs following BMT. Defective in vivo function of $\mathrm{Nrf2}^{-2^{--}}$HSPCs was linked to HSPC exhaustion as a result of poor antioxidant defenses, diminished survival signaling (24), and enhanced proliferation under steady-state conditions (55). Here, we demonstrate for what we believe to be the first time that aug- 

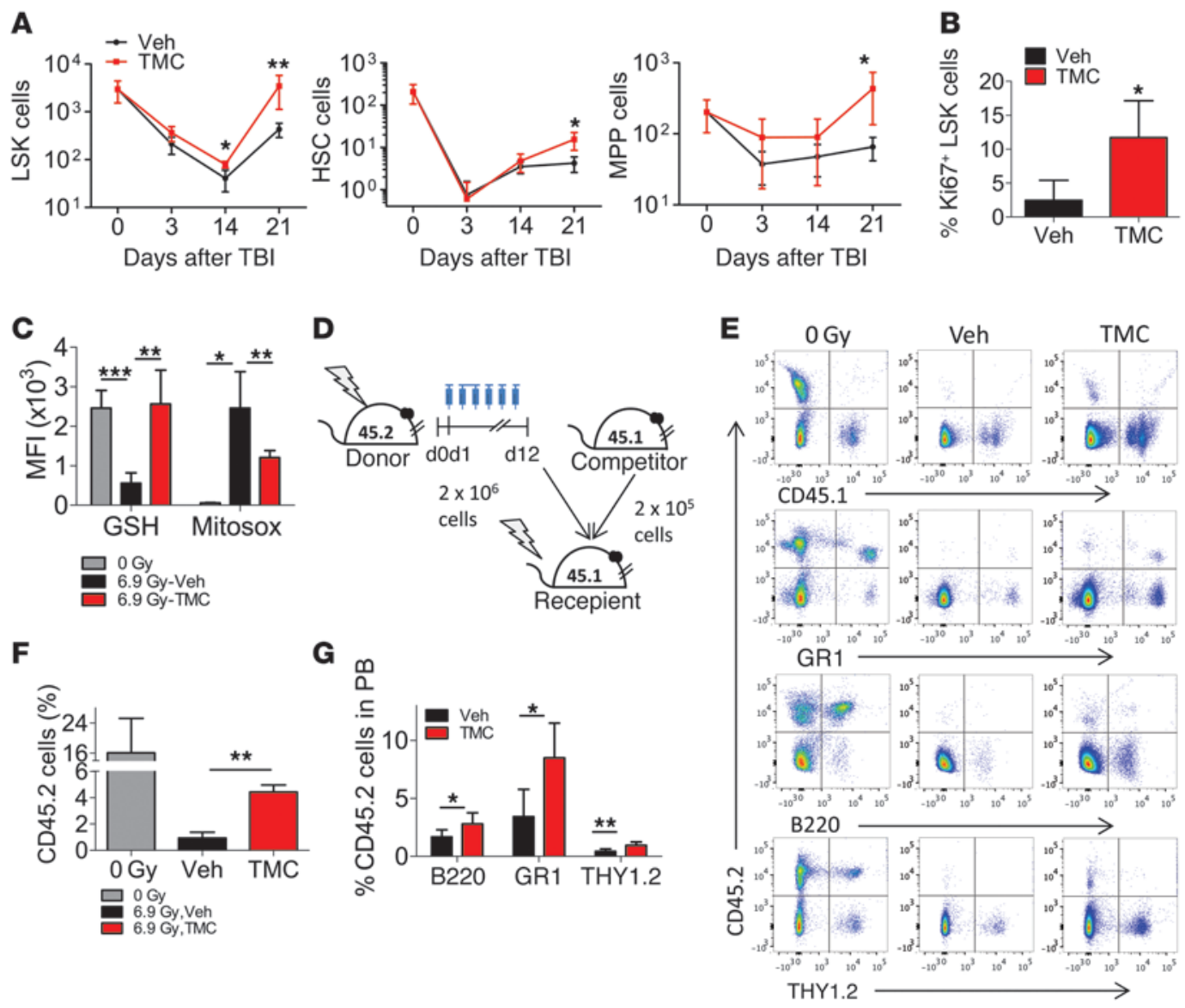

\section{Figure 6}

Administration of NRF2 activator TMC enhances expansion of HSPCs in TBI-exposed mice. (A) Analysis of LSK, HSC (LSK CD48-CD150+), and MPP (LSK CD48-CD150-) cell populations in the BM of TMC- or vehicle-administered mice on days 3,14 , and 21 after TBI. ${ }^{*} P<0.05$ and ${ }^{\star \star} P<0.01$ compared with vehicle-treated mice $(n=15)$. (B) Flow cytometric analysis of KI67 markers in LSK cells isolated from BM of TMC- or vehicle-treated mice on day 23 after TBI. Data represent the mean \pm SD of the percentage of $\mathrm{KI}_{67}{ }^{+} \mathrm{LSK}$ cells. ${ }^{*} P<0.05$ compared with vehicle-treated mice. (C) Flow cytometric analysis of GSH and ROS levels in LSK cells isolated from TMC- or vehicle-treated mice on day 3 after TBI. Data represent the MFI \pm SD. ${ }^{*} P<0.05$ compared with vehicle-treated mice. ${ }^{* *} P<0.01$; ${ }^{\star * \star} P<0.001$. (D) Schematic of experiments depicted in E-G. BM cells isolated from vehicle- or TMC-treated mice 24 hours after the last dose (day 12) was injected into lethally irradiated recipient mice together with competitive CD45.1 cells. (E and F) Flow cytometric analysis showing percentage of donor-derived CD45.2 PB cells in recipient mice 6 weeks after BMT. (G) Percentage of donor CD45.2 cell-derived granulocyte (GR1 $\left.{ }^{+}\right)$, B cell (B220+), and T cell (THY1.2+) lineages in the PB of recipient mice 6 weeks after BMT. ${ }^{*} P<0.05 ;{ }^{* \star} P<0.01$.

menting NRF2 activity by genetic disruption of KEAP1 in hematopoietic stem cells and/or BM stromal cells supports the repopulating capacity of HSPCs via intrinsic and extrinsic mechanisms.

Timely stimulation of HSPC proliferation is critical to early hematopoietic recovery and survival following TBI $(56,57)$. Consistent with a previous study by McDonald et al. (58), we found that lethal IR exposure caused greater mortality and hematopoietic toxicity in $\mathrm{Nrf2} 2^{-/-}$mice than in $\mathrm{Nrf2} 2^{+/+}$mice, indicating the protective role of NRF2 in radiation-induced myelosuppression. NRF2-deficient cells are shown to be sensitive to radiation-induced DNA damage, ROS, and cell death $(58,59)$. Contrary to our expectations, we observed a marked downregulation of NRF2 activity in HSPCs until day 2 that was restored to baseline levels by day 12 in $N r f 2^{+/+}$mice after TBI. In contrast, we found that BM stromal cells showed a similar decline in NRF2 activity until day 2 and then dramatic induction by day 12 after TBI. The mecha- nism for the delay in the activation of NRF2 in hematopoietic cells after TBI is unclear. Previously, McDonald et al. (58) also reported a marked delay in activation of NRF2 in cells exposed to IR, and it coincided with the rise in ROS generation. In a radiological emergency scenario, it is a realistic assumption that a medical countermeasure (MCM) will likely not be administered for a minimum of 24 hours after an acute radiation disaster (10). This 24-hour treatment window adds a stringent requirement for an effective MCM to mitigate the lethal effects of myelosuppression. Here, we demonstrated that TMC, when administered 24 hours after TBI, markedly improved survival of WT mice, but not $\mathrm{Nrf} 2^{-/-}$mice, suggesting that the therapeutic efficacy of TMC is mediated selectively by activating the NRF2 pathway. We observed that TMC treatment in irradiated mice increased NRF2-regulated antioxidants in BM cells and other radiationsensitive tissues such as small intestine. This increase in anti- 
A
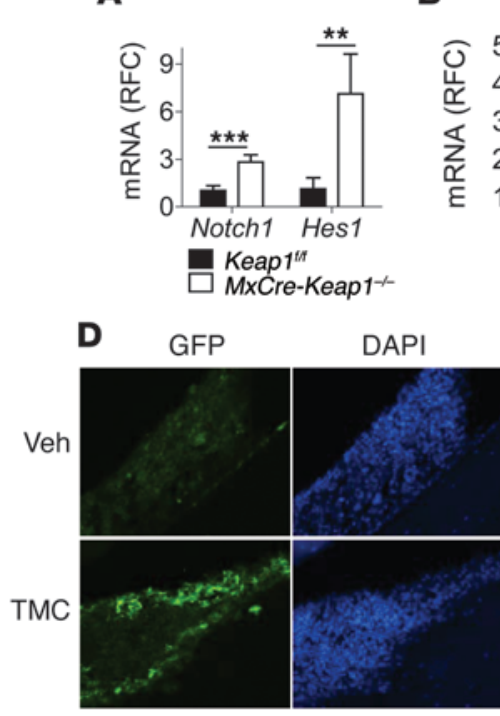

E

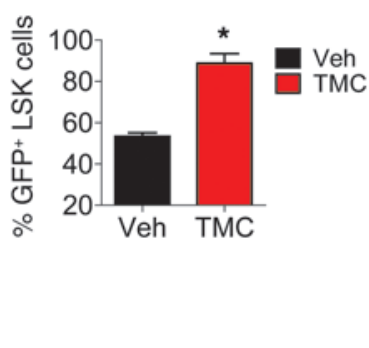

\section{Figure 7}

Administration of NRF2 activator TMC expands HSPCs by activating Notch1 signaling in irradiated mice. (A) Relative mRNA expression of Notch1 and Hes1 in BM cells isolated from unirradiated $M x C r e-K e a p 1^{\text {flox/flox }}$ mice and floxed control mice following poly $(\mathrm{I}: \mathrm{C})$ treatment. ${ }^{*} P<0.01$; ${ }^{* * \star} P<0.001$. (B) Relative mRNA expression of Notch1 and Hes 1 in BM cells isolated from unirradiated mice treated with vehicle or TMC. Mice were given 6 doses (once every 48 hours) of TMC or vehicle, beginning 24 hours after TBI. ${ }^{\star} P<0.05$. (C) Jag1 mRNA expression in BM cells isolated from vehicleor TMC-treated mice on days $2,7,13$, and 20 following TBI. ${ }^{*} P<0.05$ and ${ }^{* *} P<0.01$ compared with day- 0 vehicle-treated mice ( 0 Gy radiation). (D and E) Immunohistochemical analysis of GFP expression in bone sections (D) and GFP+ LSK cells (E) in the BM of irradiated (6.9 Gy) TNR mice treated with vehicle or TMC beginning 24 hours after TBI (6 times, once every 48 hours) on day 14 . (F) Absolute number of LSK cells in the BM of irradiated mice treated with TMC, vehicle, and/or GSI on day 12 following TBI. Mice were injected with 4 doses ( 1 dose/day) of GSI, beginning 24 hours after the first dose of TMC. ${ }^{*} P<0.05$ ( $\mathbf{D}$ and $\left.\mathbf{E}\right)$.

oxidant defenses in multiple organs may have contributed to improvement in the survival of TMC-treated mice after TBI. Nevertheless, TMC treatment 24 hours after TBI markedly enhanced hematological recovery and reduced blood bacteremia compared with vehicle-treated mice. Temporal PB and BM analysis showed that TMC treatment did not protect from early loss of circulating blood or HSCs; rather, it enhanced BM regeneration following TBI. In agreement with this, the percentage of live LSK cells was modestly elevated after treatment with TMC compared with vehicle treatment on day 3 , even though TMC treatment elevated antioxidants and reduced ROS levels in LSK cells after TBI. In contrast, we found that the total number of LSK cells was markedly elevated by day 14 after TBI. In further support, we found greater engraftment of donor BM cells isolated from TMCtreated irradiated mice than was observed in vehicle-treated mice in a competitive transplantation experiment.

Notch signaling regulates cell fate decisions by controlling self-renewal and inducing differentiation $(49,60)$. Recent findings suggest a complex role of Notch signaling in HSPC expansion and maintenance. In knockout mouse models of Notch1 or Notch2, canonical Notch signaling has been shown to be dispensable for HSC maintenance (61). Conversely, activation of Notch signaling was shown to promote HSPC proliferation $(62,63)$. Ectopic expression of Notch 1 or ex vivo stimulation with the Notch ligand
JAG1 enhanced cytokine-stimulated proliferation and survival of HSCs ex vivo and in vivo $(62,63)$. In our study, we found that TMC treatment induced an approximately 3 -fold increase in LSK cell proliferation in irradiated mice compared with that in vehicle-treated mice. Previously, we reported that NRF2 regulates transcriptional expression of Notch1 (41). Activation of NRF2 promoted liver regeneration by activating Notch signaling (41). We elucidated that TMC improved hematopoietic regeneration by upregulating Notch signaling in irradiated mice through activation of NRF2. In the present study, we found that compared with the respective control mice, LSK cells isolated from MxCre-Keap $1^{-/-}$or TMC-treated irradiated mice showed elevated expression of Notch1 and Hes1. TMC treatment induced a 2-fold increase in Notch-activated LSK cells $\left(\mathrm{GFP}^{+}\right.$cells) in irradiated TNR mice compared with that observed in vehicle-treated mice. Conversely, we found that the Notch inhibitor GSI abrogated TMC-induced improvement in hematopoietic regeneration in irradiated mice, suggesting that TMC improved hematopoietic regeneration by activating the Notch signaling cascade.

JAG1 expressed by BM stromal cells interacts with Notch-expressing hematopoietic cells and stimulates expansion and survival of HSCs (64). Recently, by global ChIPSeq analysis, we reported that NRF2 regulates JAG1 expression in mouse embryonic fibroblast cells (42); however, the biological role of NRF2 in regulating HSPC function through JAG1 was not elucidated. In the present study, we found that in vivo TMC treatment markedly induced the expression of JAG1, but not of other Notch ligands, in BM stromal cells of irradiated mice). We observed that TMC exposure in in vitro cultures of BM stromal cells iso-

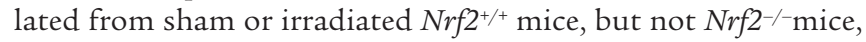
increased the induction of JAG1 expression compared with that in vehicle-treated mice and supported an enhanced ex vivo expansion of LSK cells from irradiated mice in coculture conditions. Therefore, HSPC expansion in the BM of TMC-treated irradiated mice was partly driven by NRF2-regulated JAG1/Notch1 signaling.

In conclusion, we demonstrate here a previously unknown function of NRF2 signaling in the promotion of hematopoietic reconstitution after radiation-induced myeloablation and BMT. Furthermore, we elucidate a novel mechanism in which NRF2 regulates the repopulating capacity of HSCs by activating Notch signaling via transcriptional expression of Notch1 and Jag1. Our findings suggest a translational potential of the NRF2 activator TMC for improving hematopoietic cell transplantation and mitigating radiation-induced myelosupression in a nuclear accident scenario. 

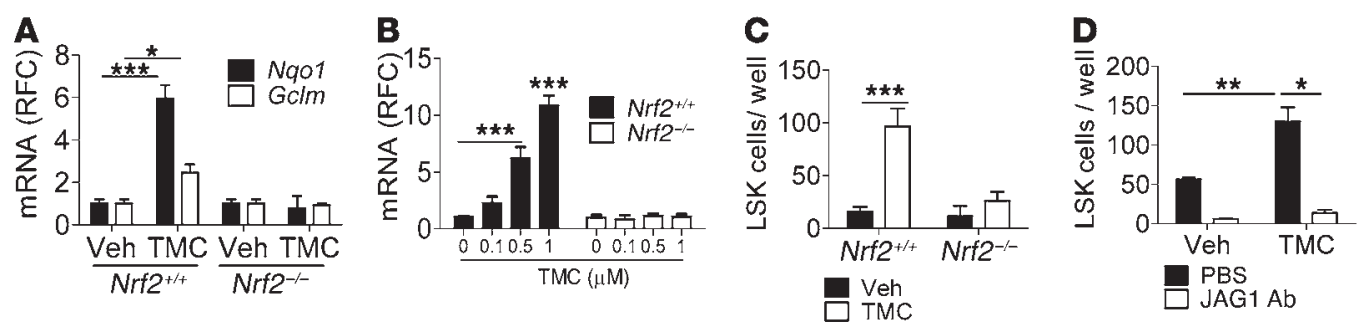

Figure 8

TMC enhanced expansion of HSPCs ex vivo by upregulating JAG1 in BM stromal cells in an NRF2-dependent manner. (A) Expression of $\mathrm{NqO1}$ and $\mathrm{Gclm}$ in BM stromal cells isolated from $\mathrm{Nrf2}^{+/+}$or $\mathrm{Nrf2}^{-/-}$unirradiated mice 12 hours after exposure to TMC $(1 \mu \mathrm{M})$ or DMSO. Data represent the mean $\pm \mathrm{SD}$. ${ }^{* \star *} P<0.001$ and ${ }^{*} P<0.05$ compared with vehicle-treated mice. (B) Jag1 mRNA expression in $\mathrm{Nrf2}^{+/+}$and $\mathrm{Nrf}^{-/-}$stromal cells isolated from BM 12 hours after exposure to increasing doses of TMC or DMSO in vitro. ${ }^{* \star *} P<0.001$. (C) LSK cells isolated from irradiated mice were cocultured with TMC- $(1 \mu \mathrm{M})$ or vehicle-exposed $\mathrm{Nrf2}^{+/+}$or $\mathrm{Nrf2}^{-/-} \mathrm{BM}$ stromal cells, and the total number of LSK cells was enumerated on day $7 .{ }^{* * *} P<0.001$. (D) LSK cells were cocultured with TMC- $(1 \mu \mathrm{M})$ or vehicle-exposed BM stromal cells in the presence or absence of anti-JAG1 antibody, and the total number of LSK cells was enumerated on day 7. LSK and BM stromal cells were isolated from irradiated mice. For neutralization of JAG1, TMC- or vehicle-treated BM stromal cells were incubated with anti-JAG1 antibody prior to incubation with CD34-LSK cells. ${ }^{*} P<0.05 ;{ }^{* *} P<0.01$.

\section{Methods}

Mice. C57BL/6, CD-1 and SJL/C57BL/6 CD45.1 mice were obtained from the National Cancer Institute (NCI) and The Jackson Laboratory, respec-

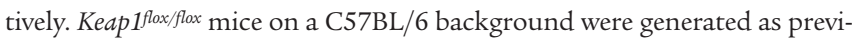
ously described $(65,66)$. MxCre-Keap $1^{\text {flox/flox }}$ mice were generated by crossing Keap $1^{\text {flox/flox }}$ mice with $\mathrm{Mx} 1-\mathrm{Cr} \mathrm{e}^{+}$mice (The Jackson Laboratory). To induce Cre-mediated deletion of Keap1 in the hematopoietic lineage, $M x$ Cre-Keap $1^{\text {flox/flox }}$ or Keap $1^{\text {flox/flox }}$ mice were administered with polyriboinosinic acid-polyribocytidylic acid [poly(I:C)] (Sigma-Aldrich) for 7 days (25 mg/kg/day) (67). Tamoxifen-inducible CMVCre-Keap $1^{\text {flox } / \text { flox }}$ mice were generated by crossing Keap $1^{f l o x} /$ flox mice with CAG-CreERT2 ${ }^{+}$mice (The Jackson Laboratory). To induce Cre-mediated deletion of Keap1, tamoxifen-inducible CMVCre-Keap $1^{\text {flox/flox }}$ or Keap $1^{\text {flox } / \text { flox }}$ mice were treated with tamoxifen for 5 days ( $1 \mathrm{mg} / \mathrm{mouse} /$ day; i.p. injection). Deletion of Keap1 exons 2-3 were confirmed by PCR analysis using primers: forward 5'-GAGTCCACAGTGTGTGGCC-3', reverse 5'-GAGTCACCGTAAGCCTGGTC-3'. Generation of NRF2-deficient C57BL/6 mice was described previously (68).

Pharmacological NRF2 activators. TMC was synthesized as previously described (28). CDDO-Me was provided by Michael B. Sporn (Dartmouth Medical School).

TBI and treatment. Mice were exposed to whole-body IR using a Gammacell 40 Exactor cesium-137 irradiator (Atomic Energy Commission of Canada, Ltd.), with an average dose rate of $0.53 \mathrm{~Gy} / \mathrm{min}^{-1}$ at the Johns Hopkins School of Medicine Radiation Oncology Department. For assessing survival rates, mice were monitored for up to 30 days after TBI. For evaluation of the radiomitigating efficacy of TMC, irradiated mice were administered orally with TMC $(10 \mathrm{mg} / \mathrm{mice} /$ day $)$ or an equal volume of vehicle 6 times, once every 48 hours, beginning 1, 6, or 24 hours after TBI. For safety studies, normal mice were administered orally with TMC or vehicle daily for 2 weeks. TMC was dissolved in a formulation consisting of 5\% DMSO and 95\% polyethylene glycol (PEG) 200. To test the radiomitigating effect of $\mathrm{CDDO}-\mathrm{Me}$, mice were treated every 48 hours with CDDO-Me or vehicle by oral gavage 1, 6, and 24 hours after TBI. CDDO-Me was dissolved in 10\% DMSO, $10 \%$ cremophor-EL, and $80 \%$ PBS. The dose of CDDO-Me was $10 \mu \mathrm{mol} / \mathrm{kg}$ body weight.

Isolation of BM cells and flow cytometry. BM cell isolation, LSK cell sorting, and flow cytometric analysis of cell surface markers were performed as previously described $(43,69)$. Briefly, BM cells were flushed from the long bones (tibias and femurs) of mice with Iscove's modified Dulbecco's that to detect biotin-conjugated lineage markers, streptavidin-conjugated APC-Cy7 (eBioscience) was used. Stained cells were analyzed using an LSR II flow cytometer (BD Biosciences). For sorting of CD34-LSK cells, FITCCD34 (eBioscience) antibody was used in addition to c-Kit, SCA1, and lineage markers. To sort live cells, 7-AAD was used and sorted by a MoFlo Sorter (Beckman Coulter). For donor-derived cell analysis in PB, rbc-free cells were stained with FITC-CD45.1, APC-CD45.2, and/or Alexa Fluor 700-CD45.2 (BD Biosciences). For analysis of multilineage blood cells, GR1-V450 (eBioscience), B220-APC-Cy7 (eBioscience), and THY1.2-APC (eBioscience) antibodies were used. To assess proliferation, LSK markers were stained with KI67-FITC or KI67-PerCPcy5.5 antibody (eBioscience). All FACS data were analyzed by FlowJo software (Tree Star Inc.). For counting BM stromal cells, PE-TER119 and APC-CD45 antibodies (eBioscience) were used.

$P B$ analysis. Complete blood counts were analyzed by a Hemavet 950FS (Drew Scientific).

$B M T$. For competitive transplantation assays, 1,000 sorted LSK cells from MxCre-Keap $1^{\text {flox/flox }}$ or Keap $1^{\text {flox } / \text { flox }}$ mice were injected retro-orbitally into lethally irradiated congenic CD45.1 ${ }^{+}$WT recipient mice with 250,000 $\mathrm{CD} 45.1^{+}$competitive cells. For the short-term repopulation assay, 500,000 whole BM cells or 1,000 donor LSK cells were injected into lethally irradiated (10 Gy) recipient mice.

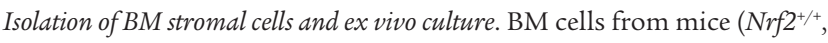
$\mathrm{Nrf2} 2^{--}$, or floxed control CMV-Keap1Cre) were collected by flushing the femurs and tibias with IMDM supplemented with 5\% FCS. BM cells that were rbc depleted were plated onto a 12 -well plate at a density of $5 \times 10^{6}$ cells per well. Culture medium was changed daily to remove the nonadherent cells, and the adherent BM stromal cells were treated with different concentrations of TMC or vehicle (DMSO) after 48 hours of culture (70). Sorted LSK cells (500-1,000 cells) were cocultured with BM stromal cells pretreated with pharmacological NRF2 activator or 4-hydroxy-tamoxifen. Culture medium containing IMDM plus $10 \%$ FBS, $1 \%$ penicillin-streptomycin, thrombopoietin, SCF, and FLT3 ligand (TSF) was prepared as previously described (43).

CFC assays. CFC assays were performed using MethoCult M3434 (STEMCELL Technologies) as previously described (24).

Quantitative real-time RT-PCR. Total RNA was extracted from cells or tissues using the RNeasy Mini Kit according to the manufacturer's protocol (QIAGEN). Quantitative real-time RT-PCR analyses were conducted using 
the assay on demand probe sets (Applied Biosystems), and reactions were analyzed using the ABI 7000 TaqMan system (Applied Biosystems) as previously described (71). GAPDH or ActB was used for normalization.

Glutathione and ROS analysis. Glutathione (GSH) and mitochondrial ROS levels were determined by flow cytometry using monochlorobimane and MitoSOX dye (Invitrogen), respectively, as previously described $(72,73)$.

Blood bacteremia. Total anticoagulated blood $(100 \mu \mathrm{l})$ from irradiated mice were cultured on LB agar or brain heart infusion agar plates (BD), as previously described $(74,75)$. After overnight incubation, bacteria colonies were counted.

Statistics. Unless otherwise noted, $P$ values were calculated using a 2 -tailed Student's $t$ test with unpaired analysis, and a $P$ value less than 0.05 was considered significant. All error bar data represent the mean \pm SD.

Study approval. Mice were housed under controlled conditions for temperature and humidity using a 12 -hour light/12-hour dark cycle. All animal experiments were performed using protocols approved by the IACUC of Johns Hopkins University.

\section{Acknowledgments}

We acknowledge the help of the Mouse Phenotyping Core, the Department of Molecular and Comparative Pathobiology, and the Flow Cytometry Core of Johns Hopkins University. We thank Michael B. Sporn (Dartmouth Medical School) for providing CDDO-Me. We wish to thank Steven Ahn (Johns Hopkins University Bloomberg School of Public Health) for editing the manuscript and Il Min (Johns Hopkins University School of Medicine) for helpful discussions. We also thank
G. Xin (Johns Hopkins University Bloomberg School of Public Health) for timely help with the mice studies and T. Kensler (Johns Hopkins University) and M. Yamamoto (Tohoku University, Aoba-ku, Sendai, Japan) for providing Nrf2-/- mice. Finally, we thank Nicholas Gaiano (Johns Hopkins University) for the Notch reporter mice. This work was supported by NIH grants R33AI080541, U01AI107361, and R01 CA140492 (to S. Biswal); a grant from the Flight Attendant Medical Research Institute (to S. Biswal and R.K. Thimmulappa); a NCI lung spore grant P50 CA058184 (to S. Biswal); and by National Institute on Environmental Health Sciences grants P50ES015903, ES03819, U01HL105569, P50HL107169, and P01ES018176. V. Kumar and S.V. Malhotra would like to acknowledge support from the NCI, NIH, under contract no. HHSN261200800001E.

Received for publication May 1, 2013, and accepted in revised form October 31, 2013.

Address correspondence to: Shyam Biswal, 615 N. Wolfe Street, Room E7624, Baltimore, Maryland 21205, USA. Phone: 410.955.4738; Fax: 410.955.0116; E-mail: sbiswal@jhsph.edu. Or to: Rajesh Thimmulappa, 615 N. Wolfe Street, Room W7035, Baltimore, Maryland 21205, USA. Phone: 410.502.1949; Fax: 410.955.0116; E-mail: rthimmul@jhsph.edu. Or to: Sanjay V. Malhotra, 1050 Boyles Street, Bldg 322, Rm 103, Frederick, Maryland 21702, USA. Phone: 301.846.5141; Fax: 301.846.5206; E-mail: malhotrasa@mail.nih.gov.
1. Williams JP, et al. Animal models for medical countermeasures to radiation exposure. Radiat Res. 2010; 173(4):557-578.

2. Johnson SM, et al. Mitigation of hematologic radiation toxicity in mice through pharmacological quiescence induced by CDK4/ 6 inhibition. J Clin Invest. 2010;120(7):2528-2536.

3. Mauch P, et al. Hematopoietic stem cell compartment: acute and late effects of radiation therapy and chemotherapy. Int J Radiat Oncol Biol Phys. 1995; 31(5):1319-1339.

4. Geiger $\mathrm{H}$, et al. Pharmacological targeting of the thrombomodulin-activated protein $\mathrm{C}$ pathway mitigates radiation toxicity. Nat Med. 2012; 18(7):1123-1129

5. Guinan EC, et al. Bactericidal/permeability-increasing protein (rBPI21) and fluoroquinolone mitigate radiation-induced bone marrow aplasia and death. Sci Transl Med. 2011;3(110):110ra118.

6 . Farese AM, et al. A nonhuman primate model of the hematopoietic acute radiation syndrome plus medical management. Health Phys. 2012; 103(4):367-382.

7. Chua HL, et al. Long-term hematopoietic stem cell damage in a murine model of the hematopoietic syndrome of the acute radiation syndrome. Health Phys. 2012;103(4):356-366.

8. Coleman CN, Stone HB, Moulder JE, Pellmar TC. Medicine. Modulation of radiation injury. Science. 2004;304(5671):693-694.

9. DiCarlo AL, Ramakrishnan N, Hatchett RJ. Radiation combined injury: overview of NIAID research. Health Phys. 2010;98(6):863-867.

10. Stone HB, et al. Models for evaluating agents intended for the prophylaxis, mitigation and treatment of radiation injuries. Report of an NCI Workshop, December 3-4, 2003. Radiat Res. 2004; 162(6):711-728.

11. Mercier FE, Ragu C, Scadden DT. The bone marrow at the crossroads of blood and immunity. Nat Rev Immunol. 2012;12(1):49-60.

12. Wang Y, Oberley LW, Murhammer DW. Evidence of oxidative stress following the viral infection of two lepidopteran insect cell lines. Free Radic Biol Med. 2001;31(11):1448-1455

13. Wang Y, Schulte BA, LaRue AC, Ogawa M, Zhou D. Total body irradiation selectively induces murine hematopoietic stem cell senescence. Blood. 2006; 107(1):358-366.

14. Burdelya LG, et al. An agonist of toll-like receptor 5 has radioprotective activity in mouse and primate models. Science. 2008;320(5873):226-230.

15. Kawakatsu M, et al. Nicaraven attenuates radiation-induced injury in hematopoietic stem/progenitor cells in mice. PLoS One. 2013;8(3):e60023.

16. Moding EJ, Kastan MB, Kirsch DG. Strategies for optimizing the response of cancer and normal tissues to radiation. Nat Rev Drug Discov. 2013; 12(7):526-542.

17. Rangasamy T, et al. Genetic ablation of Nrf2 enhances susceptibility to cigarette smoke-induced emphysema in mice. JClin Invest. 2004;114(9):1248-1259.

18. Sykiotis GP, Bohmann D. Stress-activated cap'n'collar transcription factors in aging and human disease. Sci Signal. 2010;3(112):re3.

19. Kensler TW, Wakabayashi N, Biswal S. Cell survival responses to environmental stresses via the Keap1Nrf2-ARE pathway. Annu Rev Pharmacol Toxicol. 2007; 47:89-116.

20. Kim SB, et al. Targeting of Nrf2 induces DNA damage signaling and protects colonic epithelial cells from ionizing radiation. Proc Natl Acad SciUS A. 2012; 109(43):E2949-E2955.

21. Singh A, Bodas M, Wakabayashi N, Bunz F, Biswal S. Gain of Nrf2 function in non-small-cell lung cancer cells confers radioresistance. Antioxid Redox Signal. 2010;13(11):1627-1637.

22. Travis EL, et al. NRF2 deficiency reduces life span of mice administered thoracic irradiation. Free Radic Biol Med. 2011;51(6):1175-1183.

23. Thimmulappa RK, Mai KH, Srisuma S, Kensler TW, Yamamoto M, Biswal S. Identification of Nrf2-regulated genes induced by the chemopreventive agent sulforaphane by oligonucleotide microarray. Cancer Res. 2002;62(18):5196-5203.

24. Merchant AA, Singh A, Matsui W, Biswal S. The redox-sensitive transcription factor $\mathrm{Nrf} 2$ regulates murine hematopoietic stem cell survival independently of ROS levels. Blood. 2011;118(25):6572-6579.

25. Essers MA, et al. IFNalpha activates dormant haematopoietic stem cells in vivo. Nature. 2009; 458(7240):904-908.

26. Kiel MJ, Morrison SJ. Uncertainty in the niches that maintain haematopoietic stem cells. Nat Rev Immunol. 2008;8(4):290-301.

27. Morrison SJ, Spradling AC. Stem cells and niches: mechanisms that promote stem cell maintenance throughout life. Cell. 2008;132(4):598-611.

28. Kumar V, et al. Novel chalcone derivatives as potent Nrf2 activators in mice and human lung epithelial cells. J Med Chem. 2011;54(12):4147-4159.

29. Nguyen T, Sherratt PJ, Huang HC, Yang CS, Pickett $\mathrm{CB}$. Increased protein stability as a mechanism that enhances Nrf2-mediated transcriptional activation of the antioxidant response element. Degradation of Nrf2 by the $26 \mathrm{~S}$ proteasome. J Biol Chem. 2003; 278(7):4536-4541.

30. Malhotra D, et al. Decline in NRF2-regulated antioxidants in chronic obstructive pulmonary disease lungs due to loss of its positive regulator, DJ-1. Am J Respir Crit Care Med. 2008;178(6):592-604.

31. Stewart D, Killeen E, Naquin R, Alam S, Alam J. Degradation of transcription factor $\mathrm{Nrf2}$ via the ubiquitin-proteasome pathway and stabilization by cadmium. J Biol Chem. 2003;278(4):2396-2402.

32. McMahon M, Itoh K, Yamamoto M, Hayes JD. Keap1-dependent proteasomal degradation of transcription factor Nrf2 contributes to the negative regulation of antioxidant response element-driven gene expression. J Biol Chem. 2003; 278(24):21592-21600

33. Itoh K, Wakabayashi N, Katoh Y, Ishii T, O'Connor T, Yamamoto M. Keap1 regulates both cytoplasmic-nuclear shuttling and degradation of $\mathrm{Nrf2}$ in response to electrophiles. Genes Cells. 2003; 8(4):379-391. 
34. Thimmulappa RK, et al. Preclinical evaluation of targeting the Nrf2 pathway by triterpenoids (CDDO-Im and CDDO-Me) for protection from LPS-induced inflammatory response and reactive oxygen species in human peripheral blood mononuclear cells and neutrophils. Antioxid Redox Signal. 2007;9(11):1963-1970.

35. Dinkova-Kostova AT, et al. Extremely potent triterpenoid inducers of the phase 2 response: correlations of protection against oxidant and inflammatory stress. Proc Natl Acad Sci U S A. 2005; 102(12):4584-4589.

36. Yates MS, et al. Pharmacodynamic characterization of chemopreventive triterpenoids as exceptionally potent inducers of $\mathrm{Nrf2}$-regulated genes. Mol Cancer Ther. 2007;6(1):154-162.

37. Pergola PE, et al. Bardoxolone methyl and kidney function in CKD with type 2 diabetes. NEngl J Med. 2011; 365(4):327-336.

38. Pergola PE, et al. Effect of bardoxolone methyl on kidney function in patients with T2D and Stage 3b-4 CKD. Am J Nephrol. 2011;33(5):469-476.

39. Zhang DD. Bardoxolone brings nrf2-based therapies to light. Antioxid Redox Signal. 2013;19(5):517-518.

40. Malhotra D, et al. Global mapping of binding sites for Nrf2 identifies novel targets in cell survival response through ChIP-Seq profiling and network analysis. Nucleic Acids Res. 2010;38(17):5718-5734.

41. Wakabayashi N, et al. Regulation of notch1 signaling by nrf2: implications for tissue regeneration. Sci Signal. 2010;3(130):ra52.

42. Malhotra D, et al. Global mapping of binding sites for Nrf2 identifies novel targets in cell survival response through ChIP-Seq profiling and network analysis. Nucleic Acids Res. 2010;38(17):5718-5734.

43. Himburg HA, et al. Pleiotrophin regulates the expansion and regeneration of hematopoietic stem cells. Nat Med. 2010;16(4):475-482.

44. Real PJ, et al. Gamma-secretase inhibitors reverse glucocorticoid resistance in $\mathrm{T}$ cell acute lymphoblastic leukemia. Nat Med. 2009;15(1):50-58.

45. Stier S, Cheng T, Dombkowski D, Carlesso N, Scadden DT. Notch 1 activation increases hematopoietic stem cell self-renewal in vivo and favors lymphoid over myeloid lineage outcome. Blood. 2002; 99(7):2369-2378.

46. Trowbridge JJ, Xenocostas A, Moon RT, Bhatia M. Glycogen synthase kinase- 3 is an in vivo regulator of hematopoietic stem cell repopulation. Nat Med. 2006; 12(1):89-98

47. Varnum-Finney B, et al. Pluripotent, cytokine-dependent, hematopoietic stem cells are immortalized by constitutive Notch 1 signaling. Nat Med. 2000;
6(11):1278-1281.

48. Burns CE, Traver D, Mayhall E, Shepard JL, Zon LI. Hematopoietic stem cell fate is established by the Notch-Runx pathway. Genes Dev. 2005; 19(19):2331-2342.

49. Duncan AW, et al. Integration of Notch and Wnt signaling in hematopoietic stem cell maintenance. Nat Immunol. 2005;6(3):314-322.

50. Karanu FN, et al. The notch ligand jagged-1 represents a novel growth factor of human hematopoietic stem cells. J Exp Med. 2000;192(9):1365-1372.

51. Calvi LM, et al. Osteoblastic cells regulate the haematopoietic stem cell niche. Nature. 2003; 425(6960):841-846

52. Doan PL, et al. Epidermal growth factor regulates hematopoietic regeneration after radiation injury. Nat Med. 2013;19(3):295-304.

53. Ito K, et al. Regulation of oxidative stress by ATM is required for self-renewal of haematopoietic stem cells. Nature. 2004;431(7011):997-1002.

54. Suzuki T, Motohashi H, Yamamoto M. Toward clinical application of the Keap1-Nrf2 pathway. Trends Pharmacol Sci. 2013;34(6):340-346.

55. Tsai JJ, et al. Nrf2 regulates haematopoietic stem cell function. Nat Cell Biol. 2013;15(3):309-316.

56. Farese AM, Herodin F, McKearn JP, Baum C, Burton E, MacVittie TJ. Acceleration of hematopoietic reconstitution with a synthetic cytokine (SC-55494) after radiation-induced bone marrow aplasia. Blood. 1996;87(2):581-591.

57. MacVittie TJ, Farese AM, Smith WG, Baum CM, Burton E, McKearn JP. Myelopoietin, an engineered chimeric IL-3 and G-CSF receptor agonist, stimulates multilineage hematopoietic recovery in a nonhuman primate model of radiation-induced myelosuppression. Blood. 2000;95(3):837-845.

58 . McDonald JT, et al. Ionizing radiation activates the Nrf2 antioxidant response. Cancer Res. 2010; 70(21):8886-8895.

59. Kim SB, et al. Targeting of Nrf2 induces DNA damage signaling and protects colonic epithelial cells from ionizing radiation. Proc Natl Acad Sci U S A. 2012;109(43):E2949-2955.

60. Kopan R, Ilagan MX. The canonical Notch signaling pathway: unfolding the activation mechanism. Cell. 2009;137(2):216-233.

61. Varnum-Finney B, Halasz LM, Sun M, Gridley T, Radtke F, Bernstein ID. Notch2 governs the rate of generation of mouse long- and short-term repopulating stem cells. J Clin Invest. 2011;121(3):1207-1216.

62. Butler JM, et al. Endothelial cells are essential for the self-renewal and repopulation of Notch-dependent hematopoietic stem cells. Cell Stem Cell. 2010;
6(3):251-264

63. Mancini SJ, Mantei N, Dumortier A, Suter U, MacDonald HR, Radtke F. Jagged1-dependent Notch signaling is dispensable for hematopoietic stem cell self-renewal and differentiation. Blood. 2005; 105(6):2340-2342.

64. Jones P, et al. Stromal expression of Jagged 1 promotes colony formation by fetal hematopoietic progenitor cells. Blood. 1998;92(5):1505-1511.

65. Kong X, et al. Enhancing Nrf2 pathway by disruption of Keap 1 in myeloid leukocytes protects against sepsis. Am J Respir Crit Care Med. 2011; 184(8):928-938.

66. Blake DJ, et al. Deletion of Keap1 in the lung attenuates acute cigarette smoke-induced oxidative stress and inflammation. Am J Respir Cell Mol Biol. 2010; 42(5):524-536.

67. Yilmaz $\mathrm{OH}$, et al. Pten dependence distinguishes haematopoietic stem cells from leukaemia-initiating cells. Nature. 2006;441(7092):475-482.

68. Sussan TE, et al. Targeting Nrf2 with the triterpenoid CDDO-imidazolide attenuates cigarette smoke-induced emphysema and cardiac dysfunction in mice. Proc Natl Acad Sci U S A. 2009; 106(1):250-255

69. Mayle A, Luo M, Jeong M, Goodell MA. Flow cytometry analysis of murine hematopoietic stem cells. Cytometry A. 2013;83(1):27-37.

70. Soleimani M, Nadri S. A protocol for isolation and culture of mesenchymal stem cells from mouse bone marrow. Nat Protoc. 2009;4(1):102-106.

71. Thimmulappa RK, et al. Nrf2-dependent protection from LPS induced inflammatory response and mortality by CDDO-Imidazolide. Biochem Biophys Res Commun. 2006;351(4):883-889.

72. Kong X, Thimmulappa R, Kombairaju P, Biswal S. NADPH oxidase-dependent reactive oxygen species mediate amplified TLR4 signaling and sepsis-induced mortality in Nrf2-deficient mice. J Immunol. 2010;185(1):569-577.

73. Malhotra D, et al. Denitrosylation of HDAC2 by targeting Nrf2 restores glucocorticosteroid sensitivity in macrophages from COPD patients. J Clin Invest. 2011;121(11):4289-4302.

74. Brook I, Walker RI, MacVittie TJ. Effect of radiation dose on the recovery of aerobic and anaerobic bacteria from mice. Can J Microbiol. 1986; 32(9):719-722

75. Abdallah BM, et al. dlk1/FA1 regulates the function of human bone marrow mesenchymal stem cells by modulating gene expression of pro-inflammatory cytokines and immune response-related factors. J Biol Chem. 2007;282(10):7339-7351. 